\title{
The supernova remnant W44: Confirmations and challenges for cosmic-ray acceleration
}

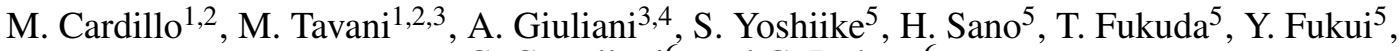 \\ G. Castelletti ${ }^{6}$, and G. Dubner ${ }^{6}$
}

1 INAF/IAPS, 00133 Roma, Italy e-mail: mcardillo@roma2.infn.it

2 Dip. di Fisica, Univ. Tor Vergata, 00133 Roma, Italy

3 CIFS-Torino, 10133 Torino, Italy

4 INAF/IASF-Milano, 20133 Milano, Italy

5 Department of Physics and Astrophysics, Nagoya University, Furo-cho, Chikusa-ku, Nagoya 464-8601, Japan

${ }^{6}$ Instituto de Astronomía y Física del Espacio (IAFE), CC.67, Suc.28, 1428 Buenos Aires, Argentina

Received 16 September 2013 / Accepted 5 March 2014

\begin{abstract}
The middle-aged supernova remnant (SNR) W44 has recently attracted attention because of its relevance regarding the origin of Galactic cosmic-rays. For the first time for a SNR, the gamma-ray missions AGILE and Fermi have established the spectral continuum below $200 \mathrm{MeV}$, which can be attributed to a neutral pion emission. Confirming the hadronic origin of the gamma-ray emission near $100 \mathrm{MeV}$ is then of the greatest importance. Our paper is focused on a global re-assessment of all available data and models of particle acceleration in W44 with the goal of determining the hadronic and leptonic contributions to the overall spectrum on a firm ground. We also present new gamma-ray and CO NANTEN2 data on W44 and compare them to recently published AGILE and Fermi data. Our analysis strengthens previous studies and observations of the W44 complex environment and provides new information for more detailed modeling. In particular, we determine that the average gas density of the regions emitting $100 \mathrm{MeV}-10 \mathrm{GeV}$ gammarays is relatively high $\left(n \sim 250-300 \mathrm{~cm}^{-3}\right)$. The hadronic interpretation of the gamma-ray spectrum of W44 is viable and supported by strong evidence. It implies a relatively large value for the average magnetic field $\left(B \geq 10^{2} \mu \mathrm{G}\right)$ in the SNR surroundings, which is a sign of field amplification by shock-driven turbulence. Our new analysis establishes that the spectral index of the proton energy distribution function is $p_{1}=2.2 \pm 0.1$ at low energies and $p_{2}=3.2 \pm 0.1$ at high energies. We critically discuss hadronic versus leptonic-only models of emission taking radio and gamma-ray data into account simultaneously. We find that the leptonic models are disfavored by the combination of radio and gamma-ray data. Having determined the hadronic nature of the gamma-ray emission on firm ground, a number of theoretical challenges remain to be addressed.
\end{abstract}

Key words. acceleration of particles - astroparticle physics - shock waves - radiation mechanisms: non-thermal ISM: supernova remnants - gamma rays: ISM

\section{Introduction}

Cosmic-rays (CRs) are highly energetic particles (with kinetic energies up to $E=10^{20} \mathrm{eV}$ ), which are mainly composed of protons and nuclei with a small percentage of electrons (1\%). Currently, the CR origin is one of the most important problems of high-energy astrophysics, and the issue is the subject of very intense research (Fermi 1949; Ginzburg \& Syrovatskii 1964; Berezinskii et al. 1990). For recent reviews, see Helder et al. (2012) and Aharonian (2012). Focusing on CRs produced in our Galaxy (energies up to the so-called "knee", $E=10^{15} \mathrm{eV}$ ), strong shocks in supernova remnants (SNRs) are considered the most probable CR sources (e.g., Ginzburg \& Syrovatskii 1964). This hypothesis is supported by several "indirect" signatures which indicate the presence of ultra-high energy electrons (recent review in Vink 2012). However, the final proof for the origin of CRs up to the knee can only be obtained through two fundamental signatures. The first is the identification of sources emitting a photon spectrum up to $\mathrm{PeV}$ energies. The second is the detection of a clear gamma-ray signature of $\pi^{0}$ decay in Galactic sources. Both indications are quite difficult to obtain. The "Pevatron" sources are notoriously hard to find (see
Aharonian 2012, for a review), and the neutral pion decay signature is not easy to identify because of the possible contribution from co-spatial leptonic emission. Hadronic (expected to produce the $\pi^{0}$ decay spectral signature) and leptonic components can in principle be distinguished in the $50-200 \mathrm{MeV}$ energy band, where they are expected to show different behaviors.

Over the last five years, AGILE, Fermi-LAT and ground telescopes operating in the TeV energy range (HESS, VERITAS, and MAGIC) collected a great amount of data from SNRs (Abdo et al. 2009, 2010a,b,c,e,d, 2011; Acciari et al. 2009; Tavani et al. 2010; Acciari et al. 2010, 2011; Aharonian et al. 2001, 2007, 2008; Aleksic et al. 2012; Giordano et al. 2012; Giuliani et al. 2010; Hewitt et al. 2012; Katsuta et al. 2012; Lemoine-Goumard et al. 2012), providing important information and challenging theoretical models. For example, most of the observed SNRs appear to have a spectrum that is steeper than the one expected from linear and non-linear diffusive shock acceleration models (DSA) of index near 2 (and possibly convex spectrum Bell 1987; Malkov \& Drury 2001; Blasi et al. 2005). The SNR W44 is one of the most interesting SNRs observed so far; it is a middle-aged SNR, bright at gamma-ray energies, and quite close to us. Its gamma-ray spectral index (indicative of the underlying 
proton/ion distribution in the hadronic model) is $p \sim 3$, which is in apparent contradiction with DSA models. The SNR W44 is therefore an ideal system to study CR acceleration in detail. The AGILE data analysis of this remnant provided information below $E=200 \mathrm{MeV}$ for the first time, which shows the lowenergy steepening, in agreement with the hadronic interpretation (Giuliani et al. 2011). Recently, an analysis of Fermi-LAT data confirms these results (Ackermann et al. 2013).

In this paper, we present a new analysis of AGILE data with a re-assessment of $\mathrm{CO}$ and radio data on W44. We also compare our results to those obtained from Fermi-LAT data. In Sect. 2, we summarize the most relevant facts about W44, and in Sect. 3, we present an updated view on the AGILE gamma-ray data and on the CO and radio data of this SNR. In Sect. 4, we discuss hadronic and leptonic models in light of our refined analysis. The implications of this work are discussed in Sect. 5. We provide relevant details about our modeling in the Appendices.

\section{The supernova remnant W44}

The SNR W44 is a middle-aged ( 20000 years old) SNR located in the Galactic Plane $(l, b)=(34.7,-0.4)$ at a distance $d \sim 3.1 \mathrm{kpc}$ (Clark \& Caswell 1976; Wolszczan et al. 1991, Fang et al. 2013 report 1.9 kpc.). Multiwavelength observations revealed interesting features. In the radio band, W44 shows a quasi-elliptical shell (Castelletti et al. 2007, and references therein); the radio shell asymmetry is probably due to expansion in an inhomogeneous ISM. In the northwest side of the remnant, which correlates with a peak of the radio emission, there is bright $[\mathrm{SII}]$ emission characteristic of shock-excited radiative filaments (Giacani et al. 1997). In the southeast side, instead, there is a molecular cloud (MC) complex embedded in the SNR shell that interacts with the source (Wootten et al. 1977; Rho et al. 1994). The OH maser (1720 MHz) emission detected in correspondence with the SNR/MC region, confirm their interaction (Claussen et al. 1997; Hoffman et al. 2005). Wolszczan et al. (1991) reported the discovery of the radio pulsar PSR B1853+01, which is located in the south part of the remnant and surrounded by a cometary-shaped pulsar wind nebula (PWN; Frail et al. 1996). This system, however, does not appear to be correlated with the detected gamma-ray emission. The X-ray observations of W44 by the Einstein Observatory (Watson et al. 1983) showed centrally peaked emission, which is later confirmed by Chandra data (Shelton et al. 2004).

The first report on W44 in the gamma-ray band was by Fermi-LAT (Abdo et al. 2010e) that showed a GeV emission morphology which has apparent good correlation with the radio shell. The Fermi-LAT energy power spectrum of W44 showed a prominent peak near $1 \mathrm{GeV}$ and a clear decrease at higher energies with a steep spectrum for the photon index near 3 (Abdo et al. 2010e). Early processing of Fermi-LAT data has a lowenergy threshold of $200 \mathrm{MeV}$, thus limiting its ability to identify a neutral pion signature. In addition to hadronic models is then not surprising that leptonic models predicting bremsstrahlung emission below $200 \mathrm{MeV}$ could not be excluded. The relatively large gamma-ray brightness of W44 and the good spectral capability of AGILE near $100 \mathrm{MeV}$ (Tavani et al. 2009; Vercellone et al. 2008, 2009) have stimulated a thorough investigation of this SNR with the AGILE data. The AGILE gamma-ray spectrum in the range of $50 \mathrm{MeV}$ to $10 \mathrm{GeV}$ confirms the highenergy steep slope up to $10 \mathrm{GeV}$ and, remarkably, identifies a spectral decrease below $200 \mathrm{MeV}$ for the first time, as expected from neutral pion decay (Giuliani et al. 2011, hereafter G11). In the analysis of G11, both leptonic and hadronic models were considered in fitting both AGILE and Fermi-LAT data. Proper consideration was given to the constraints derived from VLA radio data and NANTEN CO data for the ambient magnetic field and density, respectively. In G11, the best model was determined to be dominated by hadronic emission with a proton distribution of spectral index $p_{2}=3.0 \pm 0.1$ and a low-energy cut-off at $E_{\mathrm{c}}=6 \pm 1 \mathrm{GeV}$. The W44 gamma-ray morphology determined by AGILE agrees well with the emission detected by Fermi-LAT below $1 \mathrm{GeV}$. Furthermore, a correlation of gamma-ray emission with $\mathrm{CO}$ emission is observed, which indicates that most of the gamma-ray emission can be associated with the SNR/MC interaction. A possible large-scale influence of escaping particles accelerated at the W44 SNR shock was studied by Uchiyama et al. (2012), who noticed the existence of far and bright gamma-ray bright MCs. A new important contribution was recently produced by the Fermi-LAT team that revisited the gamma-ray emission from W44 (Ackermann et al. 2013, hereafter A13). This work was also motivated by the improvement in the LAT data analysis that permits a better study of the spectrum near $100 \mathrm{MeV}$ (Ackermann et al. 2012). The new gamma-ray spectrum of W44 by Fermi-LAT fully confirms the AGILE spectrum below $200 \mathrm{MeV}$ (Ackermann et al. 2013, for a comparison of AGILE and new Fermi-LAT data, see Fig. 6 in Appendix A). The analysis in A13 tends to exclude a leptonic-only contribution to the gamma-ray emission because it requires a very large density $\left(n \sim 650 \mathrm{~cm}^{-3}\right)$. Their best hadronic model with an assumed surrounding medium density $n \sim 100 \mathrm{~cm}^{-3}$ is based on a smoothed broken power-law hadronic distribution with a break energy $E_{\mathrm{br}}=22 \mathrm{GeV}$ and indices $p_{1}=2.36$ for $E<E_{\mathrm{br}}$ and $p_{2}=3.5$ for $E>E_{\mathrm{br}}$. Model parameters in A13 differ from those considered earlier in Abdo et al. (2010e). Apparently, bremsstrahlung emission is not considered to be relevant in the hadronic modeling of A13, even though this process could provide a non-negligible contribution to the gamma-ray emissivity in principle. An important feature of the SNR W44 spectrum is its slope at GeV energies: the index $p \sim 3$ is substantially steeper than the range that is plausibly expected in linear and non-linear DSA models. In Malkov et al. (2011), this spectral feature is explained by Alfvén damping in the presence of a relatively large-density medium where acceleration occurs. The W44 environment is quite challenging in its morphology and requires a reanalysis of its properties in the context of the crucial implications for the acceleration mechanism of CRs. We present here a new analysis of AGILE data with a revised assessment of the W44 surrounding environment, which is based on new CO data obtained from the NANTEN2 telescope.

\section{New AGILE data analysis}

We performed a global reassessment of the AGILE data on W44, including new gamma-ray data obtained until June 2012. The new data were obtained using the updated AGILE data archive, which is available at the ASDC site (http://www.asdc.asi. it). The analysis procedure is the same as described in $\mathrm{G} 11^{1}$, except for the map bin-size (that is now wider than before in order to make an analysis focused on extended features) and the substantially more extended observing period.

1 For more details about the background model, software and likelihood technique, see Giuliani et al. (2004, 2006); Bulgarelli et al. (2012); Chen et al. (2013). 
M. Cardillo et al.: The SNR W44

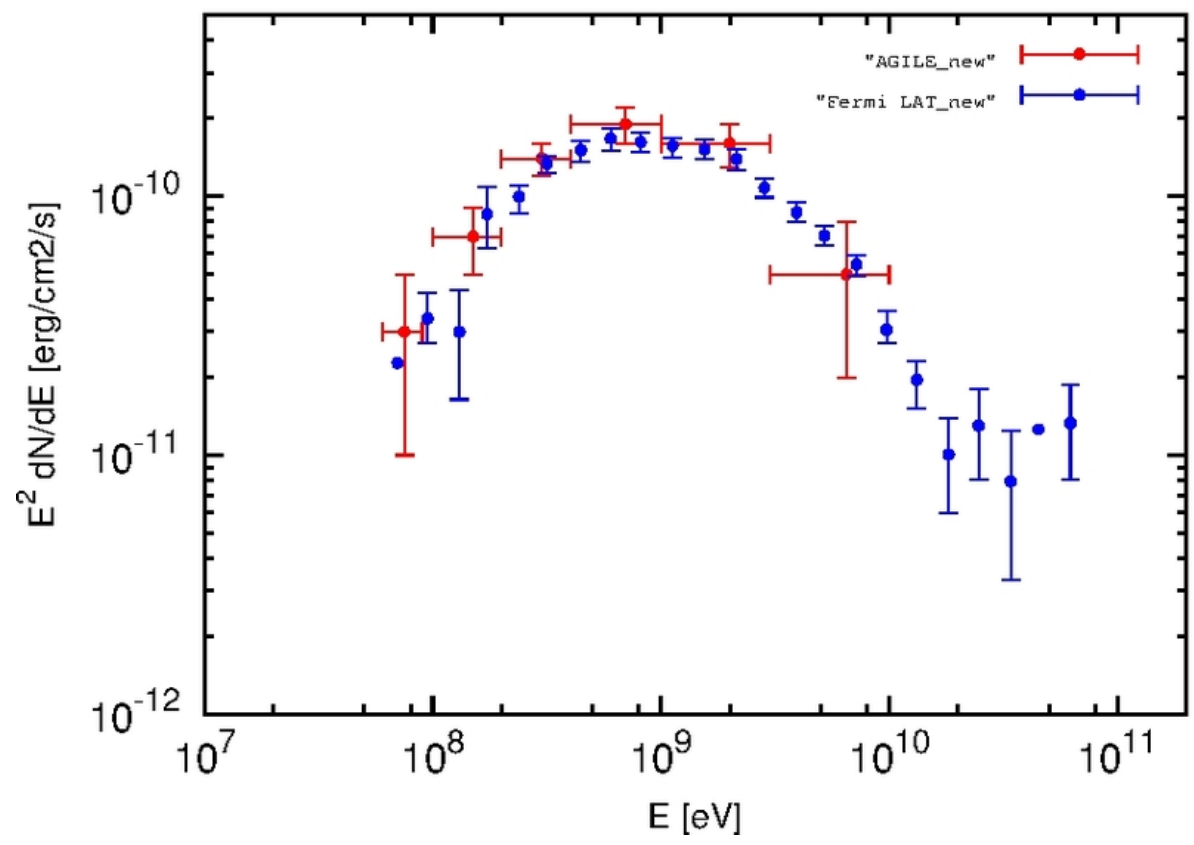

Fig. 1. AGILE new gamma-ray spectrum of SNR W44 (red data points) superimposed with the Fermi-LAT data from Ackermann et al. (2013) (blue data points).

\subsection{Morphology}

The upper left panel of Fig. 2 shows the W44 AGILE gamma-ray map in the 400-10000 MeV energy range ${ }^{2}$ with radio contours from VLA (green contour levels). The upper right panel shows the NANTEN2 telescope CO map in two velocity channels, 41 and $43 \mathrm{~km} \mathrm{~s}^{-1}$, with AGILE (magenta) and VLA (white) contours. Gamma-ray emission appears to be mostly concentrated near a high-density region, (bottom panel of the Fig. 2), indicating that most of the W44 gamma-ray emission is coincident with a site of SNR/MC interaction. This MC could be at some distance from the remnant or embedded in it. The CO maps (Fig. 2, medium panel) show the presence of a large molecular cloud complex with four different peaks at $(34.8,-0.8),(34.75,-0.5)$, $(35.1,-0.2)$, and $(34.65,-0.1)$, respectively, which are indicated by the thick white lines. Each of these peaks reaches densities of about $10^{3} \mathrm{~cm}^{-3}$ for an estimated average density in the SNR shell of $n_{\mathrm{av}} \sim 200 \mathrm{~cm}^{-3}$ (Yoshiike et al. 2013). A good correlation with the gamma-ray emission corresponds with the peak at $(34.7,-0.5)$.

\subsection{Spectrum}

Figure 1 shows the AGILE gamma-ray spectrum with the recently updated Fermi-LAT data from Ackermann et al. (2013). The AGILE spectrum is composed by six energy bins between $50 \mathrm{MeV}$ and $10 \mathrm{GeV}$ and our error-bars takes statistical errors into account ${ }^{3}$. The measured flux of the source above $400 \mathrm{MeV}$ is $F=(23 \pm 2) \times 10^{-8} \mathrm{ph} \mathrm{cm}^{-2} \mathrm{~s}^{-1}$. We notice the good agreement between the two spectra. Especially important is the confirmation of the drastic spectral decrement below $200 \mathrm{MeV}$, a crucial feature that is discussed below. Both AGILE and Fermi-LAT spectra differ from the previously published spectra in G11 and Abdo et al. (2010e) (see Appendix A).

\footnotetext{
2 The different morphology of the gamma-ray emission compared to that presented in G11 is influenced by binning. In G11, we used $0.02^{\circ} \times$ $0.02^{\circ}$ bins; instead, we make the choice of a $0.05^{\circ} \times 0.05^{\circ}$ binning.

3 The systematic errors in the canonical energy band $(100 \mathrm{MeV}-3 \mathrm{GeV})$ are on the order of $20 \%-30 \%$ of the statistical errors (Chen et al. 2013).
}

\section{Modeling}

We model the radio, AGILE, and Fermi-LAT spectral data by hadronic and leptonic-only scenarios by considering the new NANTEN2 CO data that provides a value for the ISM density in the SNR surroundings ${ }^{4}, n_{\mathrm{av}} \simeq 250 \mathrm{~cm}^{-3}$ (Yoshiike et al. 2013). This value of the average gaseous density that surrouds the gamma-ray emission is substantially larger than the one assumed in G11 and A13 $\left(n=100 \mathrm{~cm}^{-3}\right)$. Since the AGILE gammaray emission is strongly correlated with one of the $\mathrm{CO}$ peaks, we consider an average density $n \simeq 300 \pm 50 \mathrm{~cm}^{-3}>n_{\mathrm{av}}$ in the following. In modeling the spectra, we consider the most statistically significant Fermi-LAT data up to $50 \mathrm{GeV}$.

\subsection{Hadronic models}

We assume that the gamma-ray emission spectrum is due to the combined contribution of hadronic $\pi^{0}$ emission and leptonic bremsstrahlung emission by considering the proton component as the main one. For hadronic emission, we use the formalism explained in Kelner et al. (2006) that is a good approximation of the exact solution. We consider a proton distribution in total energy $E$ rather than in kinetic energy $E_{\mathrm{k}}=E-m_{\mathrm{p}} c^{2}$, following Simpson (1983) and Dermer (1986), but with $\delta$-function approximation for the cross-section (Aharonian 2004). This approximation provides that a fixed fraction of proton energy is converted to $\pi^{0}$ energy. Even if the distribution is broad, this method gives accurate results as long as the proton spectrum is smooth and broad (e.g., power-law). We fit the gamma-ray data by assuming different types of proton distributions in energy (see Fig. 3):

- a simple power-law with a high-energy cut-off (model H1):

$$
\frac{\mathrm{d} N_{p, 1}}{\mathrm{~d} E}=K_{\mathrm{p}}\left(\frac{E}{E_{\mathrm{c}}^{\mathrm{p}}}\right)^{-p_{1}} \mathrm{e}^{-\frac{E}{E_{\mathrm{c}}^{\mathrm{p}}}}
$$

4 Yoshiike et al. (2013) use a $\mathrm{H} 2 / \mathrm{CO}$ ratio which is equal to $X_{\mathrm{CO}}=$ $1.56 \times 10^{20} \mathrm{~cm}^{-2} / \mathrm{K} / \mathrm{km} \mathrm{s}^{-1}$ (see also Hunter et al. 1997) that we use as a reference value. This value of $X_{\mathrm{CO}}$ is known to be uncertain within a factor of at least 2 (see, e.g., Strong et al. 2004). 

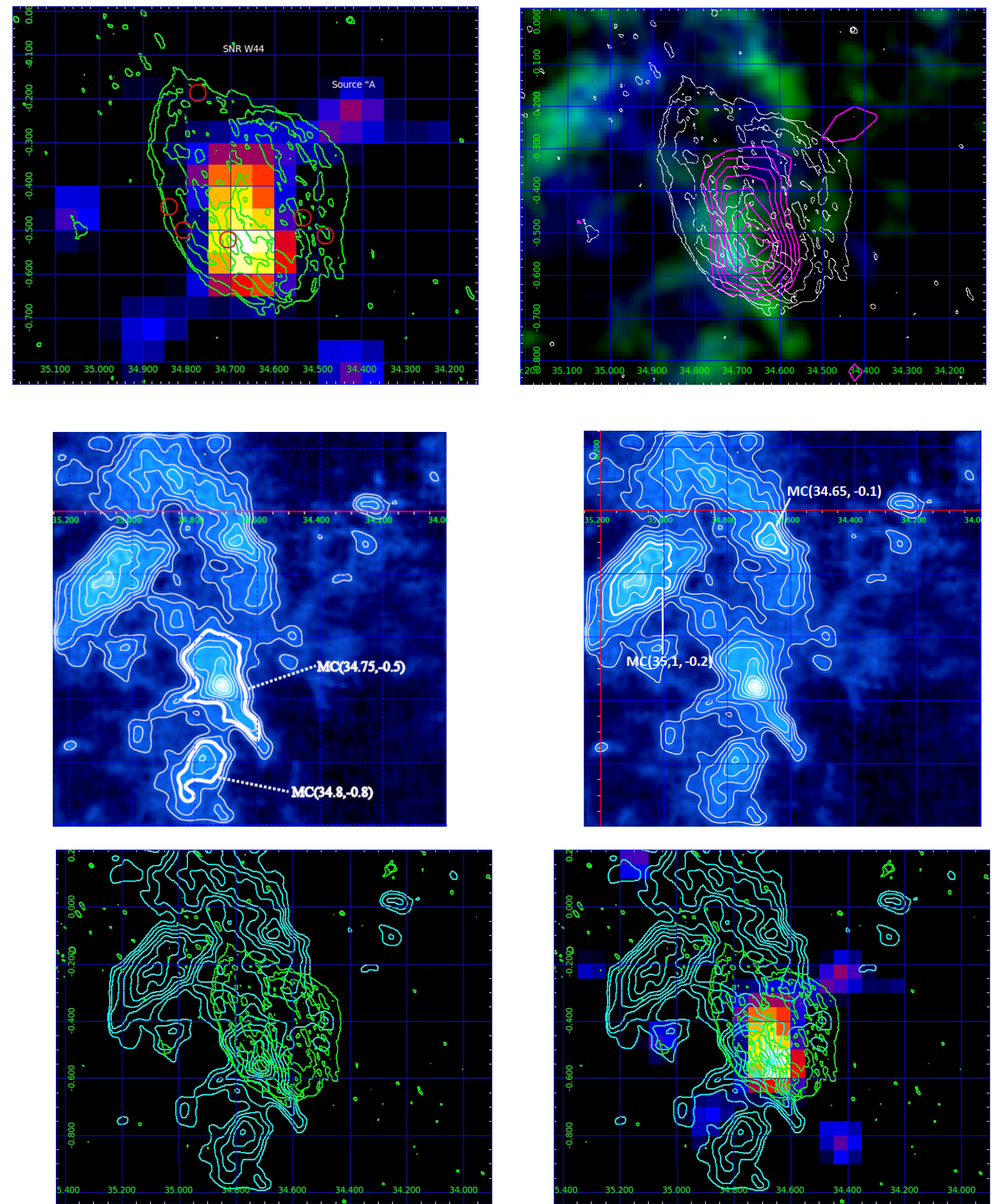

Fig. 2. Upper left panel: AGILE gamma-ray intensity map (in Galactic coordinates) of the W44 region in the energy range $400 \mathrm{MeV}-10 \mathrm{GeV}$, which is obtained by integrating all available data collected during the period from May 2007 to June 2012 . The pixel size is $0.05^{\circ} \times 0.05^{\circ}$ with a 3-bin Gaussian smoothing. Green contours show the $324 \mathrm{MHz}$ radio continuum flux density detected by the very large array (Castelletti et al. 2007) and red circles indicate detected OH masers (Claussen et al. 1997). Upper right panel: combined CO data from the NANTEN2 observatory that is superimposed with the AGILE gamma-ray data contours (magenta) above $400 \mathrm{MeV}$ of the W44 region (map in Galactic coordinates) and VLA contours (white). The CO data have been selected in the velocity range $40-43 \mathrm{~km} \mathrm{~s}^{-1}$, corresponding to a kinematic distance compatible with the W44 distance. Middle left and right panels: NANTEN2 CO integrated maps with CO contours (40-43 km s${ }^{-1}$ ). Thick white lines show the four CO peaks. Bottom left panel: NANTEN2 CO integrated contours (40-43 km s${ }^{-1}$, cyan) and VLA contours (green). Bottom right panel: AGILE intensity map (400 MeV-10 GeV) with NANTEN2 (cyan) and VLA (green) contours.

- a smoothed broken power-law (model H2):

$$
\frac{\mathrm{d} N_{\mathrm{p}, 2}}{\mathrm{~d} E}=K_{\mathrm{p}}\left(\frac{E}{E_{\mathrm{br}}^{\mathrm{p}}}\right)^{-p_{1}}\left(\frac{1}{2}\left(1+\frac{E}{E_{\mathrm{br}}^{\mathrm{p}}}\right)\right)^{p_{1}-p_{2}}
$$

- a broken power-law (model H3):

$$
\frac{\mathrm{d} N_{\mathrm{p}, 3}}{\mathrm{~d} E}= \begin{cases}K_{\mathrm{p}, 1}\left(\frac{E}{E_{\mathrm{br}}^{\mathrm{p}}}\right)^{-\mathrm{p}_{1}} & \text { if } E<E_{\mathrm{br}} \\ K_{\mathrm{p}, 2}\left(\frac{E}{E_{\mathrm{br}}^{\mathrm{p}}}\right)^{-\mathrm{p}_{2}} & \text { if } E>E_{\mathrm{br}} .\end{cases}
$$


Table 1. Hadronic model parameters.

\begin{tabular}{lccccccc}
\hline \hline Models & $p_{1}$ & $p_{2}$ & $\begin{array}{c}E_{\mathrm{br}}^{\mathrm{p}} \\
{[\mathrm{GeV}]}\end{array}$ & $\begin{array}{c}E_{\mathrm{c}}^{\mathrm{p}} \\
{[\mathrm{GeV}]}\end{array}$ & $\begin{array}{c}K_{\mathrm{p}} \\
{\left[1 / \mathrm{MeV} / \mathrm{cm}^{3}\right]}\end{array}$ & $\begin{array}{c}K_{\mathrm{e}} \\
{\left[1 / \mathrm{MeV}^{2} \mathrm{~cm}^{3}\right]}\end{array}$ & $\chi^{2} / n-1$ \\
\hline$H 1$ & $2.0 \pm 0.1$ & - & - & $45 \pm 1$ & $2 \times 10^{-14}$ & $4 \times 10^{-14}$ & 2 \\
$H 2$ & $1.7 \pm 0.1$ & $3.5 \pm 0.1$ & $16 \pm 1$ & - & $3.9 \times 10^{-14}$ & $7 \times 10^{-14}$ & 1.8 \\
$H 3$ & $2.2 \pm 0.1$ & $3.2 \pm 0.1$ & $20 \pm 1$ & - & $K_{1} \sim 1.8 \times 10^{-13}, K_{2} \sim 1.5 \times 10^{-13}$ & $1.5 \times 10^{-12}$ & 1.5 \\
\hline
\end{tabular}

Notes. $p_{1}$ is the proton spectral index before the break, $p_{2}$ is the proton spectral index above the break, $E_{\mathrm{br}}^{\mathrm{p}}$ is the proton break energy, $E_{\mathrm{c}}^{\mathrm{p}}$ is the proton cut-off energy, $K_{\mathrm{p}}$ and $K_{\mathrm{e}}$ are proton and electron normalization constants, and $\frac{\chi^{2}}{n-1}$ is the reduced chi-square.

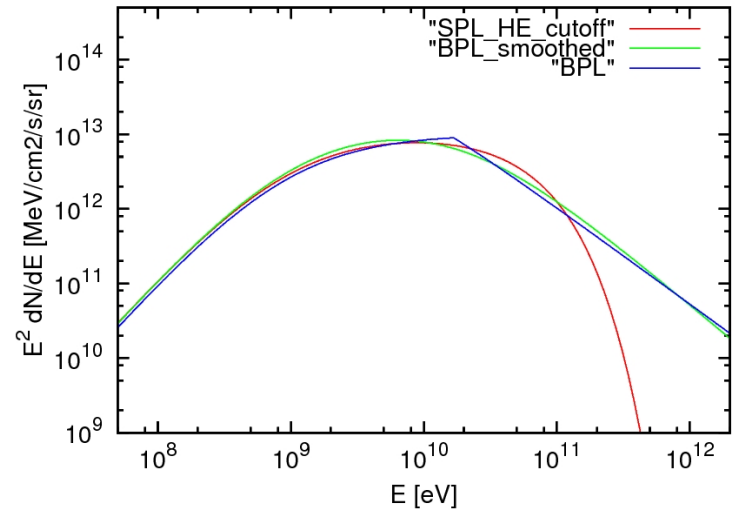

Fig. 3. Particle total energy distributions for our best hadronic models vs. the kinetic energy: a simple power-law with a high-energy cut-off at $E_{\mathrm{c}}=45 \mathrm{GeV}$ (Eq. (1), red), a smoothed broken power law with $E_{\mathrm{br}}=$ $16 \mathrm{GeV}$ (Eq. (2), green), and broken power law with $E_{\mathrm{br}}=20 \mathrm{GeV}$ (Eq. (3), blue).

For leptons, we used a simple power-law with a high energy cutoff in all hadronic models;

$$
\frac{\mathrm{d} N_{\mathrm{e}}}{\mathrm{d} E}=K_{\mathrm{e}}\left(\frac{E}{E_{\mathrm{c}}^{\mathrm{e}}}\right)^{-p^{\prime}} \mathrm{e}^{-\frac{E}{E_{\mathrm{c}}^{\mathrm{c}}}}
$$

We fix only the parameters for which we have solid observational evidence: the average medium density, $n=300 \mathrm{~cm}^{-3}$, and the radio spectral index, $p^{\prime}=1.74$. We vary all other parameters, such as the normalization constants, $K_{\mathrm{p}}$ and $K_{\mathrm{e}}$, and the cut-off and break energies, $E_{\mathrm{c}}$ and $E_{\mathrm{br}}$. Our results are summarized in Table 1, where we show the best models which are obtained according to the standard chi-square minimization test (Taylor 2000). Every model is discussed individually in Appendix B. Here, we present the properties of our best hadronic model H3. This is characterized by the distribution in Eq. (3) with $p_{1}=$ $2.2 \pm 0.1$ (for $E<E_{\mathrm{br}}$ ), $p_{2}=3.2 \pm 0.1$ (for $E>E_{\mathrm{br}}$ ), and an energy break $E_{\mathrm{br}}^{\mathrm{p}}=20 \mathrm{GeV}$. The leptonic contribution to this model is given by a simple power-law for the electrons with $p^{\prime}=1.74$, and $E_{\mathrm{c}}^{\mathrm{e}}=12 \mathrm{GeV}$ (see Fig. 4). This model provides a proton energy $W^{\mathrm{p}}=5 \times 10^{49}$ erg and requires an average magnetic field in the emission region, $B=210 \mu \mathrm{G}$.

In our calculations, we do not consider the so-called "nuclear enhancement factor" (Dermer 2012) that considers helium contribution in the gamma-ray spectrum, which is on the order of 2. However, the only change due to this factor is a reduced proton energy density; our most important results and conlusions about spectral indices and parameter estimation are not affected in any way.

\subsection{Leptonic-only models}

It is important to test the viability of leptonic-only models of gamma-ray emission from W44. We use the general expression for electron radiative processes as in Blumenthal \& Gould (1970).

- Synchrotron emission: for a power-law electron distribution, it is convenient to use a $\delta$-function approximation (e.g., Longair 2011) that can be expressed as:

$\frac{\mathrm{d} N}{\mathrm{~d} E_{\gamma}}=4 \pi \frac{1}{E_{\mathrm{ph}}} \sigma_{\mathrm{th}} U_{B} F_{\mathrm{e}}\left(E_{\mathrm{e}}\right) \mathrm{d} E_{\mathrm{e}}\left[\frac{1}{\mathrm{MeV} \mathrm{s} \mathrm{cm}^{-3}}\right]$,

where $U_{B}=\frac{B^{2}}{8 \pi} \mathrm{MeV} \mathrm{cm}^{-3}$ is magnetic energy density, $\sigma_{\text {th }}$ is Thompson cross-section, $\mathrm{d} E_{\mathrm{e}}=\frac{m_{\mathrm{e}} c^{2}}{2}\left(\frac{3}{4} \frac{1}{E_{\gamma} E_{\mathrm{ph}}}\right)$, and $E_{\mathrm{ph}}=\frac{B e}{2 \pi m_{\mathrm{e}} c} h$ is the initial photon energy. For a power-law electron distribution proportional to $E^{-p^{\prime}}$, the photon energy distribution is then proportional to $E^{-\left(\frac{p^{\prime}-1}{2}\right)}$.

- Bremsstrahlung emission: we used the general expression from Blumenthal \& Gould (1970):

$$
\begin{aligned}
& \frac{\mathrm{d} N}{\mathrm{~d} E}=\alpha r_{0}^{2} E^{-1} n \int \mathrm{d} E_{\mathrm{e}} F_{\mathrm{e}}\left(E_{\mathrm{e}}\right) E_{\mathrm{e}}^{-2} \\
& \times\left[\left(2 E_{\mathrm{e}}^{2}-2 E_{\mathrm{e}} E+E^{2}\right) \phi_{1}-\frac{2}{3} E_{\mathrm{e}}\left(E_{\mathrm{e}}-E\right) \phi_{2}\right] \\
& \times\left[\frac{1}{\mathrm{MeV} \mathrm{s} \mathrm{cm}^{-3}}\right]
\end{aligned}
$$

where $n$ is the density, $\phi_{1}$ and $\phi_{2}$ are functions of electron energies, $\alpha$ is the fine structure constant, and $r_{\mathrm{e}}$ is the electron classical radius. This can be used in both a totally ionized medium (weak shielding) and in the presence of neutrals (strong shielding); the difference is only a logarithmic factor. For a power-law electron distribution proportional to $E^{-p}$, the photon energy distribution is then proportional to $E^{-p^{\prime}}$.

- Inverse Compton emission:

$$
\frac{\mathrm{d} N}{\mathrm{~d} E}=4 \pi \frac{1}{E_{\mathrm{ph}}} \sigma_{\mathrm{kn}} U_{\mathrm{ph}} F_{\mathrm{e}}\left(E_{\mathrm{e}}\right) \mathrm{d} E_{\mathrm{e}}\left[\frac{1}{\mathrm{MeV} \mathrm{s} \mathrm{cm}^{-3}}\right],
$$

where $U_{\mathrm{ph}}$ energy density of the radiation field, $\sigma_{\mathrm{kn}}$ is Klein-Nishina cross-section, $\mathrm{d} E_{\mathrm{e}}=\frac{m_{\mathrm{e}} c^{2}}{2}\left(\frac{3}{4} \frac{1}{E_{\gamma} E_{\mathrm{ph}}}\right)$, and $E_{\mathrm{ph}}$ is the interstellar radiation field initial photon energy.

To fit both gamma-ray and radio data, we consider a smoothed broken power-law leptonic distribution:

$$
\frac{\mathrm{d} N_{\mathrm{e}, 2}}{\mathrm{~d} E}=K_{\mathrm{p}}\left(\frac{E}{E_{\mathrm{br}}^{\mathrm{e}}}\right)^{-p_{1}^{\prime}}\left(\frac{1}{2}\left(1+\frac{E}{E_{\mathrm{br}}^{\mathrm{e}}}\right)\right)^{p_{1}^{\prime}-p_{2}^{\prime}} .
$$



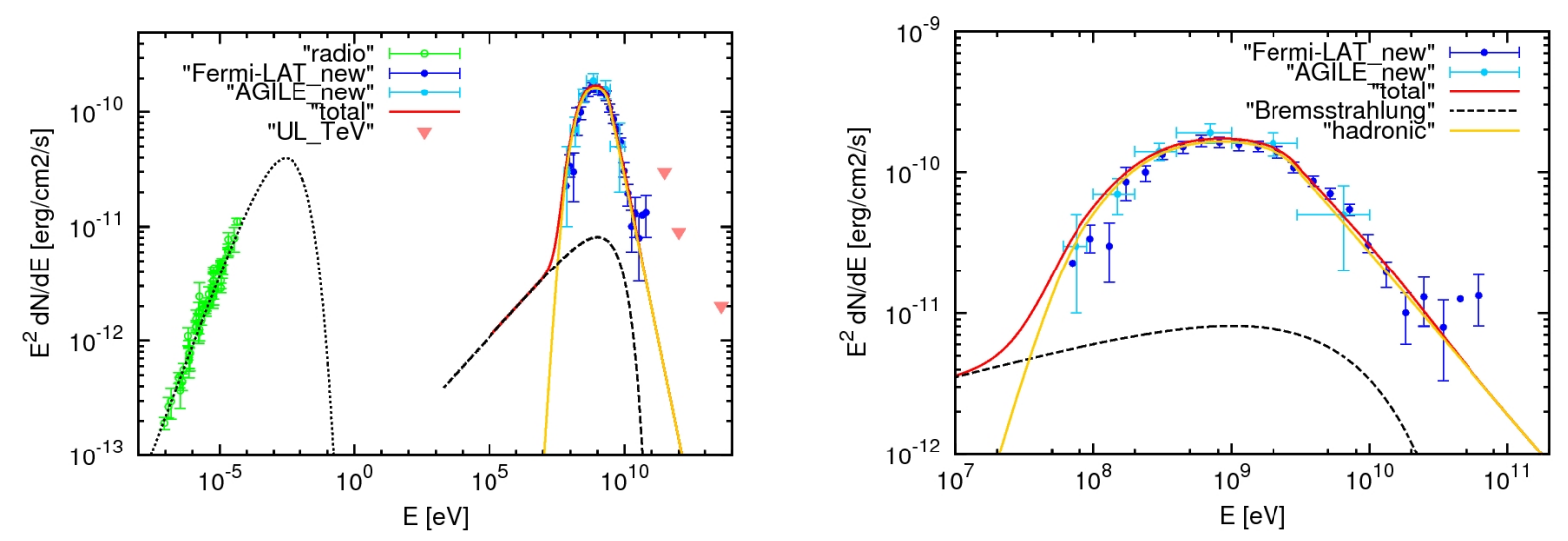

Fig. 4. Our best hadronic model, H3, of the broadband spectrum of the SNR W44 superimposed with radio (data points in green color) and gammaray data of Fig. 1 (in blue and cyan color). Proton distribution in Eq. (3) with index $p_{1}=2.2 \pm 0.1$ (for $E<E_{\mathrm{br}}$ ) and $p_{2}=3.2 \pm 0.1$ (for $E>E_{\mathrm{br}}$ ) where $E_{\mathrm{br}}^{\mathrm{p}}=20 \mathrm{GeV}$. This model is characterized by $B=210 \mu \mathrm{G}$ and $n=300 \mathrm{~cm}^{-3}$. The yellow curve shows the neutral pion emission from the accelerated proton distribution discussed in the text. The black curves show the electron contribution by synchrotron (dot) and bremsstrahlung (dashed) emissions; the IC contribution is negligible. The red curve shows the total gamma-ray emission from pion-decay and bremsstrahlung. Left panel: SED from radio to gamma-ray band. Right panel: only gamma-ray part of the spectrum.

Table 2. Leptonic model parameters.

\begin{tabular}{|c|c|c|c|c|}
\hline Models & $p_{1}^{\prime}$ & $p_{2}^{\prime}$ & $\begin{array}{c}E_{\mathrm{br}}^{\mathrm{e}} \\
{[\mathrm{GeV}]}\end{array}$ & $\begin{array}{c}K_{\mathrm{e}} \\
{\left[1 / \mathrm{MeV} / \mathrm{cm}^{-3}\right]}\end{array}$ \\
\hline L1 & 1.74 & $4.2 \pm 0.1$ & $8 \pm 1$ & $4 \times 10^{-14}$ \\
\hline $\mathrm{L} 2$ & $-2.5 \pm 0.1$ & $3.4 \pm 0.1$ & $0.5 \pm 0.1$ & $1 \times 10^{-11}$ \\
\hline
\end{tabular}

Notes. $p_{1}^{\prime}$ is the electron spectral index before the break, $p_{2}^{\prime}$ is the electron spectral index above the break, $E_{\mathrm{br}}^{\mathrm{e}}$ is the electron break energy, and $K_{\mathrm{e}}$ is the electron normalization constant.

Our assumption is that the same electron population produces both the gamma-ray and the radio fluxes through bremsstrahlung and synchrotron emissions, respectively. The spatial co-existence of radio filaments and sites of gamma-ray emission justifies this hypothesis. We fix the gaseous density value, $n=300 \mathrm{~cm}^{-3}$, from NANTEN2 data.

Our first leptonic-only model was developed (L1, see Table 2) by fixing the electron spectral index at the value found from radio data analysis by Castelletti et al. (2007); $p_{1}^{\prime}=1.74$ for $E<E_{\text {peak. }}$. We found a high energy electron spectral index $p_{2}^{\prime}=4.2 \pm 0.1$ above an energy break $E_{\mathrm{br}}^{\mathrm{e}} \sim 8 \mathrm{GeV}$ and a magnetic field $B \sim 25 \mu \mathrm{G}$. However, fixing $p_{1}^{\prime}=1.74$, we can fit radio synchrotron data, but we cannot fit it in any way the low-energy gamma-ray data (see Fig. 5).

The second leptonic-only model was developed to fit gamma-ray data with the Bremsstrahlung emission (L2, see Table 2) by changing the electron spectral index. We can fit low-energy gamma-ray data with an index $p_{1}^{\prime}=-2.5 \pm 0.1$ for $E<E_{\mathrm{br}}^{\mathrm{e}}$, which is very hard to explain. The other parameters found are $p_{2}^{\prime}=3.4 \pm 0.1$ for $E>E_{\mathrm{br}}^{\mathrm{e}}, E_{\mathrm{br}}^{\mathrm{e}} \sim 500 \mathrm{MeV}$, and $B \sim 40 \mu \mathrm{G}$.

\section{Discussion}

\subsection{Models}

Gamma-ray emission from SNRs can be produced in general by three different mechanisms: (1) relativistic bremsstrahlung from electrons interacting with surrounding medium; (2) inverse Compton emission from electrons that scatter soft photons (e.g.,s cosmic background radiation and interstellar radiation field); and (3) proton-proton interaction producing $\pi^{0}$, which subsequently decays into two gamma-ray photons. To find an unambiguous signature of accelerated hadrons in W44, we need to clearly identify these different contributions in the high energy spectrum. As in G11, we model the gamma-ray data by considering all possible emission mechanisms. We fix two important parameters obtained from radio and $\mathrm{mm}-\mathrm{CO}$ data. Multifrequency radio data (Castelletti et al. 2007) provide the radio photon in$\operatorname{dex} \alpha=0.37$, which implies an electron index $p^{\prime}=1.74$, for energies less than the synchrotron peak. By using NANTEN2 telescope data, we can also fix the SNR average density in the region of gamma-ray emission at $n_{\mathrm{av}}=300 \mathrm{~cm}^{-3}$.

\subsubsection{Leptonic-only model failure}

Our aim is to test whether a leptonic-only model can explain the gamma-ray emission from W44. We assume that the same electron population produces both the radio and the gamma-ray emissions. We assume a broken power-law electron distribution with inverse Compton and Bremsstrahlung components.

- L1 model: we use the index $p_{1}^{\prime}=1.74$ as a parameter, which is obtained from radio synchrotron data (Castelletti et al. 2007). Relativistic bremsstrahlung has the same electron index (Blumenthal \& Gould 1970). Consequently, an index $p_{1}^{\prime}=1.74$ cannot explain the low-energy gamma-ray data in any way (Fig. 5, black curve). Moreover, the relation between density and magnetic field (see Appendix C) constrains the synchrotron peak; fixing the medium density to the average value found in Yoshiike et al. (2013), $n=300 \mathrm{~cm}^{-3}$, we cannot fit in a good way the $\mathrm{W} 44$ radio emission for any magnetic field value. The best model gives a $B=25 \mu \mathrm{G}$. Changing the density value does not improve the fit.

- L2 model: in this case, we do not apply the radio constraint on the electron spectral index to fit the low-energy gamma-ray data. We find that only an index $p_{1}^{\prime}=-2.5 \pm 0.1$ can explain the gamma-ray spectrum decay at $E<E_{\mathrm{br}}^{\mathrm{e}}$ with $E_{\mathrm{br}}^{\mathrm{e}}=500 \mathrm{MeV}$ with an index $p_{2}^{\prime}=3.4 \pm 0.1$ for $E>E_{\mathrm{br}}^{\mathrm{e}}$. In this case, the W44 gamma-ray emission can be explained, 

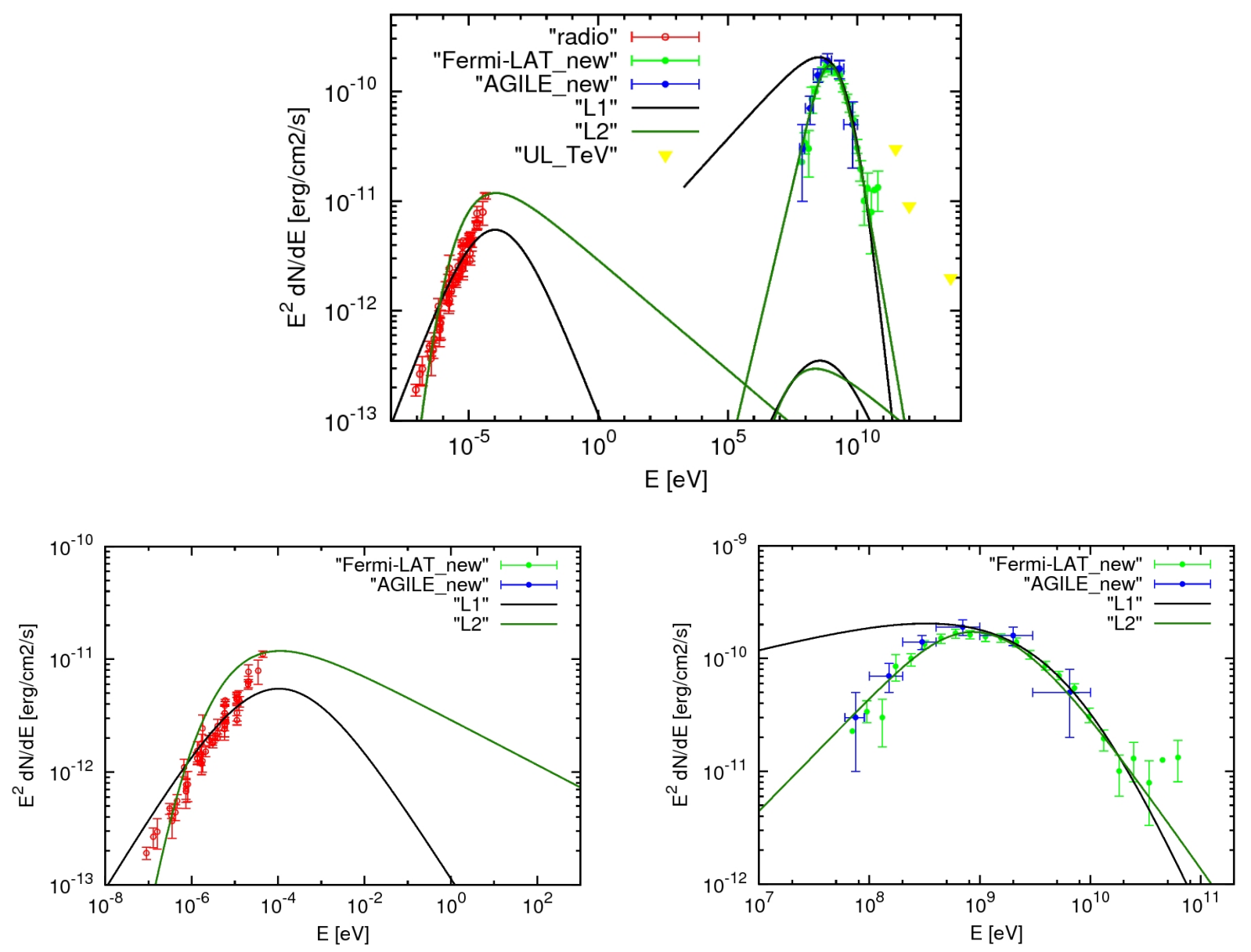

Fig. 5. Photon spectra obtained from the two leptonic-only models, L1 (green) and L2 (black), based on a broken power-law electron distribution. The density is fixed at $n=300 \mathrm{~cm}^{-3}$. In the model L1 the electron index is fixed at $p_{1}^{\prime}=1.74$, obtained from radio data, and provided the values $p_{2}^{\prime}=4.2, B=25 \mu \mathrm{G}$, and $E_{\mathrm{br}}^{\mathrm{e}}=8 \mathrm{GeV}$. In the model L2, instead, we obtained $p_{1}^{\prime}=-2.5, p_{2}^{\prime}=3.4, B=40 \mu \mathrm{G}$, and $E_{\mathrm{br}}^{\mathrm{e}}=500 \mathrm{MeV}$.

but the radio synchrotron data (see Fig. 5, green curve) contradicts with the model.

Figure 5 shows the failure of these two models ${ }^{5}, \mathrm{~L} 1$ and L2, to simultaneously explain the radio and gamma-ray emission of W44.

\subsubsection{The best hadronic model: a broken power-law distribution}

We considered different possible hadronic models (Table 1 and Fig. 3). The best one is the so-called H3, a broken power-law proton distribution discussed in the following. Features and implications of the other models, $\mathrm{H} 1$ and $\mathrm{H} 2$, are discussed in Appendix A. To separately consider the low and high energy parts of the spectrum, we consider a non-smoothed broken power-law distribution (Eq. (3)). In this way, we can study which kind of processes affect one or the other part of the spectrum. We obtain a good model with $p_{1}=2.2 \pm 0.1$ for $E<E_{\mathrm{br}}$

\footnotetext{
5 A substantial inverse Compton contribution can be easily excluded. If the soft photon source is the cosmic background radiation (CBR), we expect a second peak in the gamma-ray spectrum $\left(E_{\text {peak }} \sim 1 \mathrm{TeV}\right)$, which contradicts upper limits from Cherenkov telescopes (Abdo et al. 2010e). If soft photons come from the interstellar radiation field (ISRF), we cannot fit synchrotron radio data in any way, using reasonable magnetic field values.
}

and $p_{2}=3.2 \pm 0.1$ for $E>E_{\mathrm{br}}$, where $E_{\mathrm{br}}=20 \mathrm{GeV}$. The magnetic field is on the same order as for other hadronic models, $B=210 \mu \mathrm{G}$, and the electron/proton number ratio is $\mathfrak{R}=0.01$ at $e \sim 10 \mathrm{GeV}$. The index found for the low-energy part agrees with the one found by A13. On the other hand, the high energy index of the H3 model is substantially harder than in A13 and steeper than the one found in our previous paper, $p_{2}=3.0 \pm 0.1$ (Giuliani et al. 2011). Our analysis confirms that the SNR W44 has a gamma-ray spectral index near $10 \mathrm{GeV}$ that is steeper than all other middle-aged SNRs. Interestingly, the low-energy index near 2.3 is close to the value found in several other young SNRs (Abdo et al. 2010a; Acciari et al. 2010, 2011; Aharonian et al. 2001; Giordano et al. 2012; Hewitt et al. 2012). This can have a profound reason, and it may be related with a universal or quasiuniversal injection of energetic particles by a SNR shock.

\subsection{Hadronic models: proton energies and magnetic fields}

An important physical consequence of hadronic models is the value of the total energy going into accelerated protons. Considering the total particle energy, Castelletti et al. (2007) provided a minimum value for the total CR energy and for the magnetic field in W44, which is estimated from the radio data, assuming particle and magnetic energy equipartition; $U_{\min }=5.8 \times 10^{49} \mathrm{erg}$ and $B_{\min }=13 \mu \mathrm{G}$. For the equipartition assumption, $U_{\mathrm{min}}=2 U_{B}=2 U_{\mathrm{CR}}$. Consequently, far 
A\&A 565, A74 (2014)

Table 3. Summary of the model parameters used to fit gamma-ray and radio W44 data.

\begin{tabular}{|c|c|c|c|c|c|c|c|c|c|c|c|c|}
\hline Models & $\begin{array}{l}\langle B\rangle \\
{[\mu G]}\end{array}$ & $\begin{array}{c}\langle n\rangle \\
{\left[\mathrm{cm}^{-3}\right]}\end{array}$ & $\begin{array}{c}E_{\mathrm{br}}^{\mathrm{p}} \\
{[\mathrm{GeV}]}\end{array}$ & $\begin{array}{c}E_{\mathrm{c}}^{\mathrm{p}} \\
{[\mathrm{GeV}]}\end{array}$ & $\begin{array}{c}E_{\mathrm{br}}^{\mathrm{e}} \\
{[\mathrm{GeV}]}\end{array}$ & $\begin{array}{c}E_{\mathrm{c}}^{\mathrm{e}} \\
{[\mathrm{GeV}]}\end{array}$ & $p_{1}$ & $p_{2}$ & $p_{1}^{\prime}$ & $p_{2}^{\prime}$ & $\begin{array}{c}W_{\mathrm{p}} \\
{[\mathrm{erg}]}\end{array}$ & $\begin{array}{c}W_{\mathrm{e}} \\
{[\mathrm{erg}]}\end{array}$ \\
\hline Giuliani et al. (2011) & 70 & 100 & - & $5.5 \pm 1(\mathrm{LE})$ & - & $15 \pm 1$ & $3.0 \pm 0.1$ & - & 1.74 & - & $3.3 \times 10^{49}$ & $2.8 \times 10^{48}$ \\
\hline Ackermann et al. (2013) & - & 100 & $22 \pm 1$ & - & - & & $2.36 \pm 0.05$ & $3.5 \pm 0.3$ & - & - & $4 \times 10^{49}$ & - \\
\hline H1 & 210 & 300 & - & $45 \pm 1$ (HE) & - & $20 \pm 1$ & $2.0 \pm 0.1$ & - & 1.74 & - & $1.2 \times 10^{50}$ & $6.4 \times 10^{47}$ \\
\hline H2 & 210 & 300 & $16 \pm 1$ & - & - & $15 \pm 1$ & $1.7 \pm 0.1$ & $3.5 \pm 0.1$ & 1.74 & - & $1.3 \times 10^{49}$ & $9.6 \times 10^{48}$ \\
\hline H3 & 210 & 300 & $20 \pm 1$ & - & - & $12 \pm 1$ & $2.2 \pm 0.1$ & $3.2 \pm 0.1$ & 1.74 & - & $5 \times 10^{49}$ & $5.6 \times 10^{47}$ \\
\hline L1 & 25 & 300 & & & $8 \pm 1$ & - & - & - & 1.74 & $4.2 \pm 0.1$ & - & $3.2 \times 10^{48}$ \\
\hline L2 & 40 & 300 & - & - & $0.5 \pm 0.1$ & - & - & - & $-2.5 \pm 0.1$ & $3.4 \pm 0.1$ & - & $6.6 \times 10^{47}$ \\
\hline
\end{tabular}

Notes. $\langle B\rangle$ is the average magnetic field, $\langle n\rangle$ is the average density, $E_{\mathrm{br}}^{\mathrm{p}}$ and $E_{\mathrm{br}}^{\mathrm{e}}$ are the proton and electron break energies, $E_{\mathrm{c}}^{\mathrm{p}}$ and $E_{\mathrm{c}}^{\mathrm{e}}$ are the proton and electron cut-off energies, $p_{1}$ and $p_{2}$ are the proton indices below and above the break, $p_{1}^{\prime}$ and $p_{2}^{\prime}$ are the electron indices below and above the break, $W_{\mathrm{p}}$ and $W_{\mathrm{e}}$ are the proton and electron total energies.

away from equipartition, magnetic energy should be greater than particle energy or vice versa (Longair 2011). We can use the equipartition values for magnetic and particle energies found by Castelletti et al. (2007) to expose a contradiction in their relation. Our models, except for the simple power-law with a high energy cut-off, provide a total energy in accelerated protons that is lower than the one calculated with the equipartition assumption, which implies magnetic fields with higher values. It is essential to obtain a good estimate of the magnetic field in regions of interest in W44. To fit the gamma-ray data in our models, we also explicitly consider the bremsstrahlung contribution by electrons. This approach provides a constraint on the magnetic field. Assuming that synchrotron and bremsstrahlung emissions are originated by the same electron population, both processes depend on the electron density; the higher its value, the higher their emissivity. Consequently, to obtain a small bremsstrahlung contribution to the gamma-ray emission with a fixed target density, the magnetic field has to be enhanced in order to obtain the correct synchrotron emission and viceversa. The final result is that we can fit the radio data by only considering a magnetic field, $B \sim 10^{2} \mu \mathrm{G} \gg B_{\min }$ (see Table 3), regardless of the hadronic model. This implies that the magnetic energy should be the main contribution to the total energy. Consequently, our model $\mathrm{H} 1$ can be excluded because both magnetic and particle energies are greater than the equipartition values. Large values of the magnetic field $(B \sim 0.2 \mathrm{mG})$ in $\mathrm{W} 44$ were deduced by Claussen et al. (1997) in regions near the detected OH masers. Interestingly, from Fig. 2, we find that the gamma-ray emission detected by AGILE overlaps with one of the $\mathrm{OH}$ maser regions. To obtain a large local magnetic field (i.e., substantially larger than the equipartition one), an efficient amplification mechanism must be operating. A possible mechanism was discussed by Schure et al. (2012): a linear magnetic instability can provide the condition $\delta B \sim B_{0}$. However, it is required that the instability continues to grow in order to explain magnetic fields greater than $100 \mu \mathrm{G}$. Identifying the physical mechanisms for magnetic field amplification can be challenging. It is also important to explain the relation between magnetic field and density structures (e.g., Fig. 2 in Schure et al. 2012). In young SNRs where the ISM density is low $\left(n \sim 1 \mathrm{~cm}^{-3}\right)$, high magnetic fields are usually correlated with optical and radio filaments. In middle-aged SNRs, such as W44, which are surrounded by high ISM densities $\left(n \sim 10^{2} \mathrm{~cm}^{-3}\right)$, the magnetic field is relatively large on wider scales.

\subsection{Spectral index}

Our best hadronic model is obtained from a non-smoothed broken power-law distribution. At low-energies, we can fit the gamma-ray data with a proton distribution index $p_{1}=2.2$. This value apparently agrees with the behavior seen in younger SNRs. The difference is that in young SNRs this spectral index also applies at higher energies not being affected by propagation and damping mechanisms. On the contrary, in the middle-aged SNR W44, we find a proton index $p_{2}=3.2$ at higher energies, that is substantially steeper that the value expected from theoretical models without damping. Malkov et al. (2011) explained the W44 steep spectral index with the mechanism of Alfvèn damping, which provides a steepening of exactly one unit. However, Alfvèn damping, if it occurs in W44, cannot be acting across all the proton spectrum because the deduced low-energy index seems unaffected by it.

It is interesting to compare W44 gamma-ray spectrum to the CR particle interstellar spectrum. The interstellar cosmic-ray proton index, in momentum space, is $p_{1, \mathrm{IS}}=2.5$ below $E=$ $6.5 \mathrm{GeV}$ and $p_{2, \mathrm{IS}}=2.8$ above $E=6.5 \mathrm{GeV}$ (Dermer et al. 2013); the interstellar cosmic-ray electron spectrum, instead, seems to have an index $p_{1, \mathrm{IS}}^{\prime}=1.3-1.6$ below a few $\mathrm{GeV}$ and $p_{2, \text { IS }}^{\prime}=2.1-2.3$ above GeV energies (Strong et al. 2011, and references therein). Considering our best hadronic model, $\mathrm{H} 3$, a proton index at the lowest energies, $p_{1}=2.2$, in the energy space is provided. In the momentum space, the high energy proton spectral index, $p_{2}=3.2$, results to be steeper than the interstellar CR spectrum. For the electrons, instead, radio data provided an index $p^{\prime}=1.74$ (Castelletti et al. 2007), which is steeper than $p_{1, \mathrm{IS}}^{\prime}$ but harder than $p_{2, \mathrm{IS}}^{\prime}$. Consequently, the CR spectral behavior in SNR W44 is different from that of the interstellar $\mathrm{CR}$ spectrum. This challenging issue requires a deeper analysis beyond the scope of this paper. We also note, however, that proton and electron spectra have different indices in the interstellar case as well as in W44.

Our spectral indices are compatible with the values, $p_{1}=$ $2.36 \pm 0.05$ for $p<p_{\mathrm{br}}=22 \mathrm{GeVc}^{-1}$ and $p_{2}=3.5 \pm 0.3$ for $p>p_{\mathrm{br}}$, found in Ackermann et al. (2013), where a smoothed broken power-law is used and he electron contributions are not considered. Fang et al. (2013), instead, assume that the W44 spectral behavior is explained by diffusive shock acceleration with ion-neutral damping and consider a lower distance of the remnant $(d \sim 1.9)$ and a lower density $\left(n \sim 10^{2} \mathrm{~cm}^{-3}\right)$. In this way, they found every low magnetic field $(B \sim 10 \mu \mathrm{G})$ and a very steep spectral index, $p_{1} \sim 4.1$. A direct comparison with our results is not so trivial because of the different approaches: we, like Ackermann et al. (2013), begin from fitting our data and then we look for a physical explanation. Fang et al. (2013), instead, begin from the issue that a linear DSA theory can explain Fermi-LAT data and then obtain the parameters that are inconsistent with values from our work and Ackermann et al. (2013). 
It is interesting to remark here that data collected from the young SNRs, Tycho, and Cas A (Abdo et al. 2010a; Giordano et al. 2012) show a spectral index in the range $p_{1} \approx 2.2-2.4$, which is steeper than the value $p_{1}=2$ predicted by idealized theoretical models (assumed by Malkov et al. 2011). Other non-linear mechanisms modifying standard DSA, such as neutral leakage (Blasi et al. 2012, and references therein) or reacceleration (Blasi \& Amato 2012, and references therein), may substantially affect SNRs in the whole range of energies.

\section{Conclusions}

The SNR W44 is a crucial source providing important information about the CR origin in our Galaxy. However, several characteristics of this SNR, which have been deduced by a multifrequency approach (gamma-ray spectral indices, large magnetic field), are challenging. As discussed in this paper, W44 is a relatively close and quite bright gamma-ray source. Therefore, an excellent characterization of its gamma-ray spectrum in the range 50-200 MeV has been possible because of the good statistics achieved by AGILE and Fermi-LAT. In this paper, we reanalyzed the spectral properties and the likelihood of interpreting the decrement below $200 \mathrm{MeV}$ as a "pion bump". We performed a re-analysis of the AGILE data, by revisiting radio and $\mathrm{CO}$ data of W44. We showed the unlikeliness of leptoniconly models in their most natural form: electron distributions constrained by radio data cannot fit the broad-band W44 spectrum. On the other hand, we find that both gamma-ray and radio data can be successfully modeled by different kinds of hadronic models (H1, H2, and H3).

Our results regarding the spectral properties of the accelerated proton/ion population by the W44 shock qualitatively agree with the results of Giuliani et al. (2011). We provided a broader discussion of alternatives in this paper, and specified the role played by leptons alone and jointly with protons. In what follows, we summarize the most important physical characteristics of this source.

- Neutral pion signature: W44 is the first SNR clearly showing the so-called "pion bump" that we expect at $E \geq 67 \mathrm{MeV}$ from $\pi^{0}$-decay photons. The low-energy gamma-ray spectral index in our best model is $p_{1}=2.2 \pm 0.1$. This value is similar to those found in young SNRs, which indicates that the proton injection spectrum is affected by non-standard mechanisms of acceleration.

- High density of the surrounding environment: we determined that the average density in the W44 shell is $n_{\mathrm{av}} \sim 300 \mathrm{~cm}^{-3}$ with $n \geq 10^{3} \mathrm{~cm}^{-3}$, which corresponds with $\mathrm{CO}$ peaks (see medium panels in Fig. 2). This feature was also found in other middle-aged SNRs, like W51c and IC443 (Koo et al. 2010; Castelletti et al. 2011) and explains the high gammaray flux detected from these sources. In the SNR W28, the average density is lower, $n_{\mathrm{av}} \approx 5 \mathrm{~cm}^{-3}$ (Gabici et al. 2009), but gamma-ray emission was detected and correlates with the two MC complexes where $n \approx 10^{3} \mathrm{~cm}^{-3}$ (Giuliani et al. 2010).

- High magnetic field: in W44 our best hadronic models imply a magnetic field $B \geq 100 \mu \mathrm{G}$, which is lower than the post-shock magnetic field estimated from Zeeman splitting in the $\mathrm{OH}$ masers by Claussen et al. (1997), and substantially higher than the equipartition magnetic field (Castelletti et al. 2007). However, in most of SNRs, magnetic field estimations give values $B \sim 10-10^{2} \mu \mathrm{G}$ that are much higher than the average diffuse galactic value (e.g., see Morlino \& Caprioli 2012 for Tycho, Koo et al. 2010 for W51c, and Tavani et al. 2010 for IC443). This is hardly surprising since magnetic field compression due to the shock interaction with the ISM leads to its amplification. We need to then consider a nonlinear scenario with a back-reaction of the accelerated particle at the shock (Bell et al. 2001). The large value for the magnetic field in W44 may be linked to the environment density value, $n_{\mathrm{av}} \sim 300 \mathrm{~cm}^{-3}$ given by NANTEN2. For a lower density value, we notice that we can enhance the electron density and make plausible a lower magnetic field.

- Steepness of the high energy index: as in Abdo et al. (2010e), G11, and A13, W44 shows a spectral index $p_{2} \sim 3$ for energies above $1 \mathrm{GeV}$, that is steeper than the values found in other middle-aged SNRs. Alfvèn damping in a dense environment (Malkov et al. 2011) is a mechanism for explaining this behavior, but other possibilities exist (e.g., Blasi \& Amato 2012; Blasi et al. 2012). This is a point requiring deeper investigations in the future.

Our final conclusion is that W44 stands out as a crucial SNR whose gamma-ray emission can be firmly demonstrated to be of hadronic origin. A complete understanding of the W44 features requires modeling physical processes beyond DSA. Future investigations will have to address these issues, as well as understanding W44 within the context of other SNRs.

Acknowledgements. We thank the anonymous referee for his/her comments that led to substantial improvements of our paper. We are pleased to thank F. Aharonian for extensive discussions that stimulated parts of the work presented in this paper. Research partially supported by the ASI grants no. I/042/10/0 and I/028/12/0 and Argentina ANPCyT and CONICET grants: PICT 0902/07, 0795/08, 0571/11, PIP 2166/08, 0736/12.

\section{Appendix A: Old and new AGILE and Fermi-LAT data on W44}

Figure 6 shows the old and new AGILE and Fermi-LAT spectral gamma-ray data W44. Low-energy spectral points have been added to the Fermi-LAT spectrum because of its recently improved analysis (Ackermann et al. 2012). We notice that the lowenergy Fermi-LAT spectrum below $200 \mathrm{MeV}$ has changed with respect to the previous Fermi-LAT data of Abdo et al. (2010e). On the other hand, the AGILE data re-analyzed in this paper are not different from those previously presented in G11, except the lowest energy point between 50-100 MeV. This lowest-energy spectral data is lower than the one found in our previous paper and agrees with the Fermi-LAT spectral trend. Constraining the gamma-ray spectrum near $50 \mathrm{MeV}$ is very important with regard to a possible bremsstrahlung contribution.

\section{Appendix B: Other hadronic models}

\section{Simple power-law with a high-energy cut-off}

Following Aharonian (2004), we fit our W44 spectral data with a simple power-law with a high-energy cut-off. In this case, our best-fitting parameters are an index $p_{1}=2.0 \pm 0.1$, and a cut-off energy at $E_{\mathrm{c}}^{\mathrm{p}}=45 \mathrm{GeV}$ with $B=210 \mu \mathrm{G}$. Deduced global physical quantities are a relatively large proton energy, $W_{\mathrm{p}}=1.2 \times 10^{50} \mathrm{erg}$, and a low electron/proton energy ratio $\mathfrak{R}=0.005$. Even in this case, there are some points against the applicability of this model. First, we can only fit our data with this proton distribution by ignoring the last four Fermi 

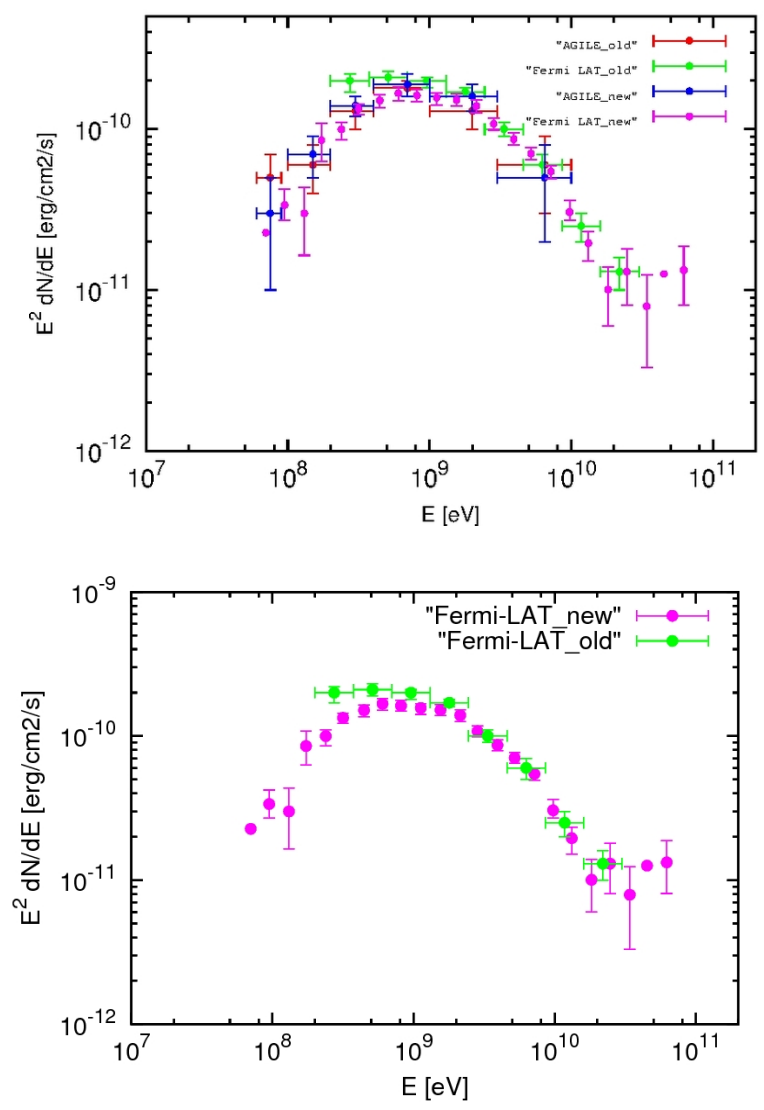

Fig. 6. Top panel: AGILE (red) and Fermi-LAT (green) old spectral energy distribution (SED) of W44 (Giuliani et al. 2011; Abdo et al. 2010e), with the new Fermi-LAT (magenta, Ackermann et al. 2013). The new AGILE data are shown in blue in Fig. 1. Bottom panel: Fermi-LAT new (magenta, Ackermann et al. 2013) and old (green, Abdo et al. 2010e) spectral energy distributions. We note that there is a substantial difference at low-energies between the two data sets.

high energy points and requiring a very low electron/proton ratio. Moreover, the high-energy cut-off considered in Aharonian (2004) for a SNR of similar age as W44 in similar density and magnetic field conditions is less sharp than the one obtained in our model, even with a high diffusion coefficient.

\section{Smoothed broken power-law}

Another way to model the W44 gamma-ray spectral data is using a smoothed broken power-law proton distribution (Eq. (2)). Our best model provides indices $p_{1}=1.74 \pm 0.1$ for $E<E_{\mathrm{br}}$ and $p_{2}=3.5 \pm 0.1$ for $E>E_{\mathrm{br}}$ with $E_{\mathrm{br}}=16 \mathrm{GeV}, B=210 \mu \mathrm{G}$, and $\mathfrak{R}=0.08$. For this proton distribution we notice that we obtain the same index of the electron distribution. In our opinion, however, the distribution of Eq. (2) cannot be considered a good model. The reason is that this model introduces a strong covariance between the low- and high-energy indices which makes their determination quite difficult and questionable. In Fig. 10 we demonstrate this point by showing the pion emission expected from the proton distribution of different indices that extend to the lowest energies and have no breaks at higher energies. The low-energy part of the spectrum of W44 can be well fit by an index in a range 2-2.3. Steeper or harder indices cannot reproduce our data, which is also true for the value $p_{1}=1.74 \pm 0.1$ found with the approach considered here.

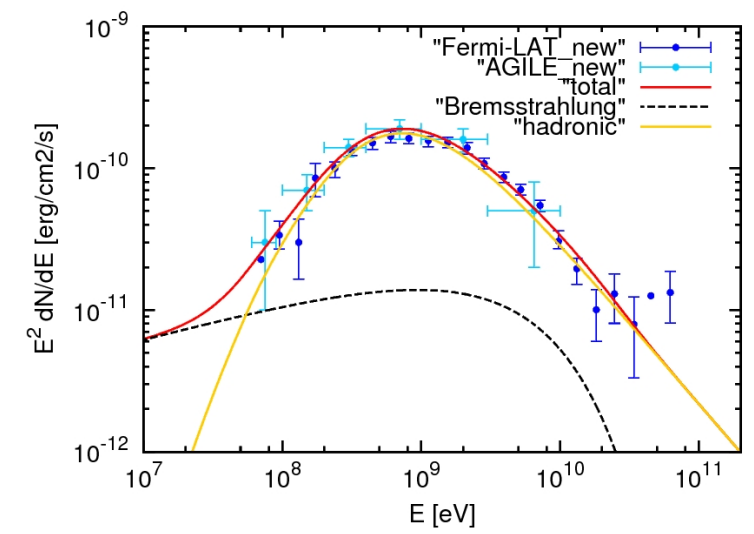

Fig. 7. Hadronic model $\mathrm{H} 1$ of the gamma-ray spectrum of W44 superimposed with the gamma-ray data of Fig. 1 (in blue and cyan colors). We find an index $p_{1}=2.0 \pm 0.1$ with a high-energy cut-off at $E_{\mathrm{c}}^{\mathrm{p}}=45 \mathrm{GeV}$. This model is characterized by $B=210 \mu \mathrm{G}$ and $n=300 \mathrm{~cm}^{-3}$. The yellow curve shows the neutral pion emission from the accelerated proton distribution discussed in the text. The dashed black curve show the electron contribution by bremsstrahlung (dashed) emissions. The IC contribution is negligible. The red curve shows the total gamma-ray emission from pion-decay and bremsstrahlung.

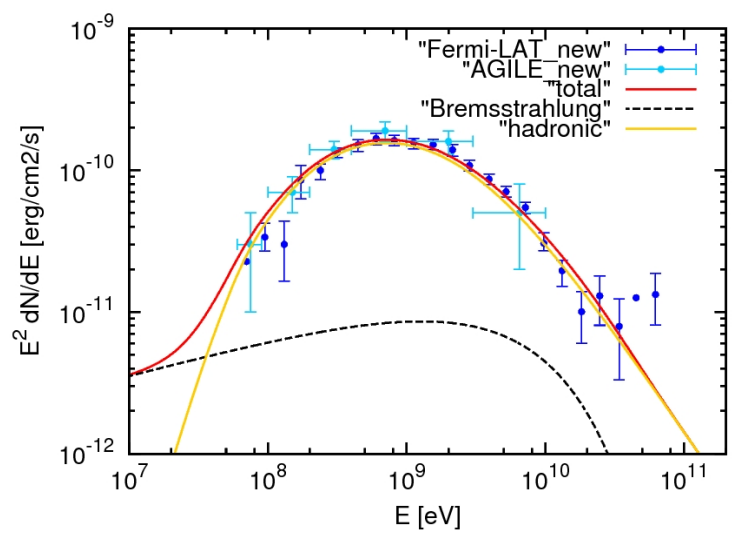

Fig. 8. Hadronic model $\mathrm{H} 2$ of the gamma-ray spectrum of W44 superimposed with the gamma-ray data of Fig. 1 (in blue and cyan colors). We find an index $p_{1}=1.74 .0 \pm 0.1$ (for $E<E_{\mathrm{br}}$ ), and $p_{2}=3.5 \pm 0.1$ (for $E>E_{\mathrm{br}}$ ) where $E_{\mathrm{br}}^{\mathrm{p}}=16 \mathrm{GeV}$. This model is characterized by $B=210 \mu \mathrm{G}$ and $n=300 \mathrm{~cm}^{-3}$. The yellow curve shows the neutral pion emission from the accelerated proton distribution discussed in the text. The dashed black curve shows the electron contribution by bremsstrahlung (dashed) emissions; the IC contribution is negligible. The red curve shows the total gamma-ray emission from pion-decay and bremsstrahlung.

\section{Simple power-law with a low-energy cut-off}

In our previous paper G11, we used a simple power-law proton distribution in kinetic energy which resulted in a spectral index $p_{1}=3.0 \pm 0.1$ and a low-energy cut off at $E_{\mathrm{k}, \mathrm{c}}^{\mathrm{p}}=5.5 \mathrm{GeV}$. We model the W44 spectral data with the same kind of distribution as in G11 here, which is in total energy rather than kinetic energy, as justified by the approach of Kelner et al. (2006):

$\frac{\mathrm{d} N_{\mathrm{p}, 4}}{\mathrm{~d} E}=K_{\mathrm{p}} E_{\mathrm{k}}^{-\mathrm{p}} \mathrm{e}^{-\frac{E_{\mathrm{k}, \mathrm{c}}^{\mathrm{p}}}{E}}$.

We obtain a reasonable good model of the spectral data with an index $p_{1}=3.2 \pm 0.1$ and a cut-off energy $E_{\mathrm{c}}^{\mathrm{p}}=6.5 \mathrm{GeV}$ for a magnetic field $B=145 \mu \mathrm{G}$ and an electron/proton energy ratio $\mathfrak{R}=W_{\mathrm{e}} / W_{\mathrm{p}}=0.03$. If the interaction of protons with 


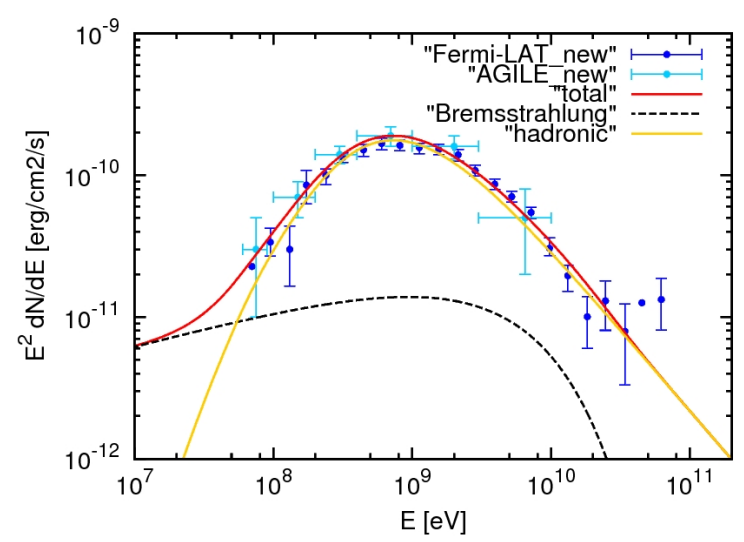

Fig. 9. Hadronic model H4 of the gamma-ray spectrum of W44 that is superimposed with the gamma-ray data of Fig. 1 (in blue and cyan colors). We find an index $p_{1}=3.2 \pm 0.1$ with a low-energy cut-off at $E_{\mathrm{c}}^{\mathrm{p}}=6.5 \mathrm{GeV}$. This model is characterized by $B=145 \mu \mathrm{G}$ and $n=300 \mathrm{~cm}^{-3}$. The yellow curve shows the neutral pion emission from the accelerated proton distribution discussed in the text. The dashed black curve show the electron contribution by bremsstrahlung (dashed) emissions; the IC contribution is negligible. The red curve shows the total gamma-ray emission from pion-decay and bremsstrahlung.

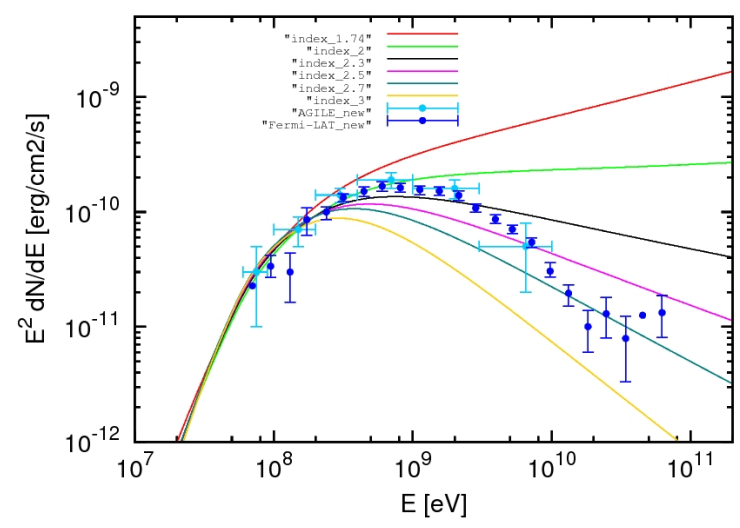

Fig. 10. Gamma-ray emission from neutral pion decay calculated for different simple power-law proton distributions of different spectral indices without any break or cut-off. We show the cases for $p_{1}=1.74$ (red), $p_{1}=2$ (green), $p_{1}=2.3$ (black), $p_{1}=2.5$ (magenta), $p_{1}=2.7$ (cyan), and $p_{1}=3$ (yellow).

the gas is outside the acceleration site, the energy-dependent diffusion of particles may produce this cutoff, as observed for the SNR W28 (Giuliani et al. 2010) and discussed by Gabici et al. (2009). Alternatively, it may be due to a suppression of the diffusion coefficient due to turbulent motions in the cloud (Gabici et al. 2007) that would exclude the low-energy CRs from the denser regions. In both cases, a slow diffusion coefficient $\left(D \sim 10^{26} \mathrm{~cm}^{2} \mathrm{~s}^{-1}\right.$ at $\left.10 \mathrm{GeV}\right)$ is required.

\section{Appendix C: Density and magnetic field link}

The relation between the magnetic field and the target density in W44 is important for our modeling. We consider here a hadronic model with the same parameters of our best model (broken power-law distribution, see Table 3) but with higher density values, $n=1000 \mathrm{~cm}^{-3}$ and $n=2000 \mathrm{~cm}^{-3}$. We assume that all gamma-ray emission detected by AGILE originates from the core of the W44 molecular cloud at Galactic coordinates $(34.75,-0.5)$. In Fig. 11 (top panel), we show the
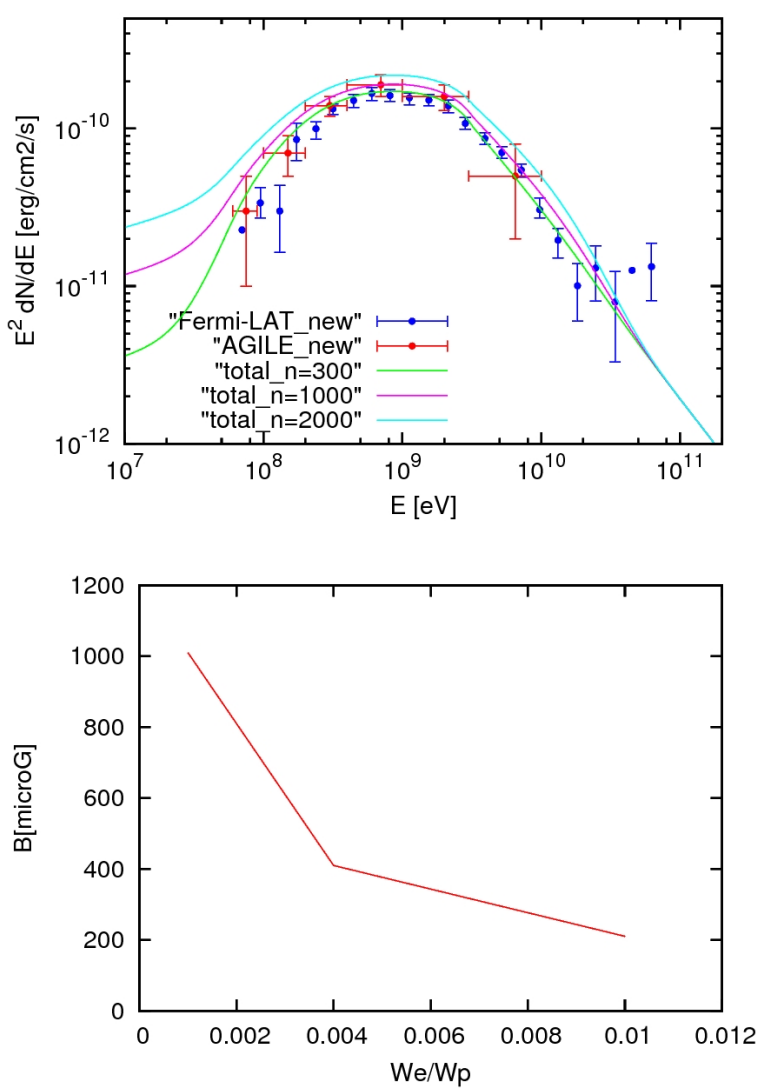

Fig. 11. Top panel: our best hadronic model H3 (see Fig. 4) for three different values of the target density; $n=300,1000$, and $2000 \mathrm{~cm}^{-3}$. Bottom panel: correlation between the magnetic field and the electron/proton ratio to fit the spectral gamma-ray data of W44 for the three assumed target densities.

results obtained with these high density values with the one obtained for $n=300 \mathrm{~cm}^{-3}$. The higher the density, the higher the bremsstrahlung contribution to the total gamma-ray emission. A very large value of the target density may lead to an overestimation of the high energy gamma-ray data. The only way to fit the data is by assuming a lower electron density (and a lower electron/proton energy ratio $\mathfrak{R}=W_{\mathrm{e}} / W_{\mathrm{p}}$ ) with a consequently higher magnetic field, $B \sim 410 \mu \mathrm{G}$ and $B \sim 1110 \mu \mathrm{G}$ for $n=1000 \mathrm{~cm}^{-3}$ and $n=2000 \mathrm{~cm}^{-3}$, respectively (Fig. 11, bottom panel).

\section{References}

Abdo, A., Ackermann, M., Ajello, M., et al. 2009, ApJ, 706, L1 Abdo, A., Ackermann, M., Ajello, M., et al. 2010a, ApJ, 710, 92 Abdo, A., Ackermann, M., Ajello, M., et al. 2010b, ApJ, 712, 459 Abdo, A., Ackermann, M., Ajello, M., et al. 2010c, ApJ, 722, 1303 Abdo, A., Ackermann, M., Ajello, M., et al. 2010d, ApJ, 718, 348 Abdo, A.,, Ackermann, M., Ajello, M., et al. 2010e, Science, 327, 1103 Abdo, A., Ackermann, M., Ajello, M., et al. 2011, ApJ, 734, 28 Acciari, V. A., Aliu, E., Arlen, T., et al. 2009, ApJ, 698, 133

Acciari, V. A., Aliu, E., Arlen, T., et al. 2010, ApJ, 714, 163

Acciari, V. A., Aliu, E., Arlen, T., et al. 2011, ApJ, 730, L720

Ackermann, M., Ajello, M., Albert, A., et al. 2012, ApJS, 203, 4

Ackermann, M., Ajello, M., Allafort, A., et al. 2013, Science, 339, 807 (A13)

Aharonian, F. A. 2001, A\&A, 370, 112

Aharonian, F. A. 2004, Very High Energy Cosmic Gamma Radiation: a Crucial

Window on the Extreme Universe (World Scientific Publishing)

Aharonian, F. 2012, Astropart. Phys., 43, 71

Aharonian, F., Akhperjanian, A., Bazer-Bachi, A., et al. 2007, A\&A, 464, 235

Aharonian, F., Akhperjanian, A., Bazer-Bachi, A., et al. 2008, A\&A, 481, 401 
Aleksic, J., Alvarez, E., Antonelli, L., et al. 2012, A\&A, 541, A13 Bell, A. R. 1978a, MNRAS, 182, 147

Bell, A. R. 1978b, MNRAS, 182, 443

Bell, A. R. 1987, MNRAS, 225, 615

Bell, A. R. 2001, MNRAS, 321, 433

Berezhko, E. G., \& Voelk, H. J. 2007, ApJ, 661, L175

Berezinskii, V. S., Bulanov, S. V., Dogiel, V. A., Ginzburg, V. L., \& Ptuskin, V. S. 1990, Astrophysics of Cosmic Rays, ed. V. L. Ginzburg (Amsterdam: North-Holland)

Blasi, P. 2012, ApJ, 755, 121

Blasi, P., \& Amato, E. 2012, JCAP, 01, 10

Blasi, P., Gabici, S., \& Vannoni, G. 2005, MNRAS, 361, 907

Blumenthal, G. R., \& Gould, R. J. 1970, Rev. Mod. Phys., 42

Bulgarelli, A., Chen, A. W., Tavani, M. V., et al. 2012, A\&A, 540, A79

Butt, Y. M. 2009, Nature, 460, 701

Castelletti, G., Dubner, G., Brogan, C., \& Kassim, N. E. 2007, A\&A, 471, 537

Castelletti, G. G., Dubner, C. T. \& Kassim, N. E. 2011, A\&A, 534, A21

Chen, A. W., Argan, A., Bulgarelli, A., et al. 2013, A\&A, 558, A37

Clark, D. H., \& Caswell, J. L. 1976, MNRAS, 174, 267

Claussen, M. J. 1999, ApJ, 522, 349

Claussen, M. J., Frail, D. A., \& Goss, W. M. 1997, ApJ, 489, 143

Dermer, C. D. 1986, A\&A, 157, 223

Dermer, C. D. 2012, Phys. Rev. Lett., 109, 091101

Dermer, C. D., Strong, A. W., Orlando, E., \& Tibaldo, L. 2013, ICRC [arXiv: 1307.0497]

Fang, J., Yu, H., Zhu, B., \& Zhang, L., et al. 2013, MNRAS, 435, 570

Fermi, E. 1949, Phys. Rev., 75, 1169

Frail, D. A., Giacani, E. B., Goss, W. M., \& Dubner, G. 1996, ApJ, 464, 165

Fukui, Y., Sano, H., Sato, J., et al. 2012, ApJ, 746, 82

Gabici, S., Aharonian, F. A., \& Blasi, P. 2007, Astrophys. Space Sci., 309, 365

Gabici, S., Aharonian, F., \& Casanova, S. 2009, MNRAS, 396, 1629

Giacani, E. B., Dubner, G., Kassim, N. E., et al. 1997, AJ, 113, 1379

Ginzburg, V. L., \& Syrovatskii, S. I. 1964, The Origin of Cosmic Rays (New York: Macmillan)

Giordano, F., Naumann-Godo, M., Ballet, K., et al. 2012, ApJ, 744, 2

Giuliani, A., Chen, A., Mereghetti, S., et al. 2004, Mem. Soc. Astron. It. Suppl., 5,135

Giuliani, A., Cocco, V., Mereghetti, S., et al. 2006, Nucl. Instrum. Methods Phys. Res. A, 568, 692

Giuliani, A., Tavani, M., Bulgarelli, A., et al. 2010, A\&A, 516, L11

Giuliani, A., Cardillo, M., Tavani, M., et al. 2011, ApJ, 742, 30 (G11)

Gomez, H. L. 2012, MNRAS, 420, 3557

Helder, E. A., Vink, J., Bykov, A. M., et al. 2012, Space Sci. Rev., 173, 369
Hewitt, J. W., Acero, E., Brandt, T. J., et al. 2012, ApJ, 759, 89

Hoffman, M., Goss, W. M., Brogan, C. L., \& Claussen, M. J. 2005, AJ, 620, 257

Hunter, S. D., Bertsch, D. L., Catelli, J. R., et al. 1997, ApJ, 481, 205

Hwang, U., \& Laming, J. M. 2012, ApJ, 746, 130

Inoue, T., Yamakazi, R., \& Inutsuka, S. 2012, ApJ, 744, 71

Katsuta, J. 2012, ApJ, 752, 135

Kelner, S. R., Aharonian, F. A., \& Bugayov, V. V. 2006, Phys. Rev. D, 74 4018

Koo, B., Heiles, C., Stanimirovic, S., \& Troland, T. 2010, AJ, 140, 262

Lemoine-Goumard, M., Renaud, M., Vink, J., et al. 2012, A\&A, 545, A28

Longair, M. 2011, High Energy Astrophysics 3rd edn. (Cambridge University Press)

Malkov, M. A., \& Drury, O. 2001, Rep. Prog. Phys., 64, 429

Malkov, M. A., Diamond, P. H., \& Sagdeev, R. Z. 2011, NatCo, 2, 194

Mori, M. 1997, ApJ, 478, 225

Morlino, G., \& Caprioli, D. 2012, A\&A, 538, A81

Onic, D. 2013, Ap\&SS, 346, 3

Picozza, P., Galper, A. M., Castellini, G., et al. 2007, Proc. ICRC

Reach, W. T., Rho, J., \& Jarret, T. H. 2005, AJ, 618, 297

Rho, J., Petre, L., Schlegel, E. M., \& Hester, J. J. 1994, AJ, 430, 757

Schure, K. M., Bell, A. R., O'C. Drury, L., \& Bykov, A. M. 2012, Space Sci. Rev., 173, 491

Shelton, R. L., Kuntz, K. D., \& Petre, R. 2004, ApJ, 611, 906

Simpson, J. A. 1983, Ann. Rev. Nucl. Part. Sci., 33, 323

Skilling, J., \& Jones, A. W. 1976, A\&A, 53, 253

Strong, A. W., Moskalenko, I. V., Reimer, O., et al. 2004, A\&A, 422, 47

Strong, A. W., Orlando, E., \& Jaffe, T. R. 2011, A\&A, 534, A54

Tavani, M., Barbiellini, G., Argan, A., et al. 2009, A\&A, 502, 995

Tavani, M., Giuliani, A., Chen, A. W., et al. 2010, ApJ, 710, 151

Taylor, J. R. 2000, An Introduction to Error Analysis: The Study of Uncertainties in Physical Measurements (University Science Books)

Torres, D. F., Romero, G. E., Dame, T. M., et al. 2003, Phys. Rep., 382, 303

Uchiyama, Y., Funk, S., Katagiri, H., et al. 2012, ApJ, 749, 35

Vercellone, S., Chen, A. W., Giuliani, A., et al. 2008, ApJ, 676, L13

Vercellone, S., Chen, A. W., Vittorini, V., et al. 2009, ApJ, 690, 1018

Vink, Y. 2012, A\&AR, 20, 49

Watson, M. G., Willingale, R., Pye, J. P., et al. 1983, Supernova remnants and their X-ray emission, Proc. Symp. (Dordrecht: D. Reidel Publishing Co), 101, 273

Wolszczan, A., Cordes, J. M., \& Dewey, R. J. 1991, AJ, 372, L99

Wootten, H. A. 1977, ApJ, 216, 440

Yoshiike, S. 2013, ApJ, 768, 179 